\title{
Prevención de infecciones asociadas a la atención de salud: conocimientos y prácticas en médicos residentes
}

\section{Prevention of health care-associated infections: knowledge and practices in resident physicians}

\author{
Martin Yagui Moscoso ${ }^{1,2, a}$, Margot Vidal-Anzardo 2,b , Leonardo Rojas Mezarina ${ }^{1,2, c}$, Hernán Sanabria Rojas ${ }^{1,2, d}$ \\ ${ }^{1}$ Facultad de Medicina, Universidad Nacional Mayor de San Marcos. Lima, Perú. \\ ${ }^{2}$ Instituto Nacional de Salud. Lima, Perú. \\ ${ }^{a}$ Médico, ORCID: https://orcid.org/0000-0002-3737-5709 \\ ${ }^{\mathrm{b}}$ Médico, ORCID: $h$ ttps://orcid.org/0000-0002-3075-7755 \\ ${ }^{\circ}$ Médico, ORCID: https://orcid.org/0000-0003-0293-7107 \\ ${ }^{\mathrm{d}}$ Médico, ORCID: https://orcid.org/0000-0003-0838-4064
}

An Fac med. 2021;82(2):131-9. / DOI: https://doi.org/10.15381/anales.v82i2.19839

\begin{abstract}
Correspondencia:
Martin Javier Alfredo Yagui Moscoso myaguim@unmsm.edu.pe
\end{abstract}

Recibido: 13 de abril 2021 Aprobado: 18 de julio 2021

Publicación en línea: 18 de agosto 2021

Conflictos de interés: Los autores declaran no tener conflictos de interés.

Fuente de financiamiento: Autofinanciado

Citar como: Yagui M, Vidal-Anzardo M, Rojas L, Sanabria H. Prevención de infecciones asociadas a la atención de salud: conocimientos y prácticas en médicos residentes. An Fac med. 2021;82(2):131-9. DOl: https://doi. org/10.15381/anales.v82i2.19839

\section{Resumen}

Objetivos. Determinar el nivel de conocimientos y prácticas sobre las medidas de prevención de las infecciones asociadas a la atención de salud (IAAS) y sus factores asociados en médicos residentes ingresantes 2018 de una universidad de Lima, Perú. Métodos. Estudio transversal en el cual se aplicó un cuestionario validado a 171 médicos residentes. Las variables estudiadas fueron edad, sexo, tiempo como profesional, capacitaciones previas, procedencia, tipo de universidad de procedencia, entre otras. Resultados. $52 \%$ de los participantes fueron de sexo femenino, el $49 \%$ tenía 2 o menos años de tiempo como profesional médico, el $49 \%$ provenía de una universidad del interior del país, solo el $34 \%$ ocupó una vacante cautiva y el $71,9 \%$ tuvo capacitaciones previas sobre medidas de prevención de IAAS. El $83 \%$ de los médicos residentes ingresantes estudiados mostró poco conocimiento sobre medidas de prevención de las IAAS mientras que el 68,4\% mostró prácticas no adecuadas sobre medidas de prevención de las IAAS. El ingreso a una especialidad de medicina estuvo asociado a un menor riesgo de tener poco conocimiento (OR: 0,32, IC 95\%: 0,11-0,93). El mayor tiempo como profesional se relacionó a un menor riesgo de tener prácticas inadecuadas (OR: 0,936, IC 95\%: 0,89-0,99). Conclusiones. Existe un bajo nivel de conocimientos sobre medidas de prevención de IAAS y una elevada proporción de los residentes ingresantes 2018 tuvo prácticas no adecuadas sobre medidas de prevención de IAAS. Se observó que el tipo de especialidad fue un factor asociado al nivel de conocimientos y el tiempo de experiencia profesional fue un factor asociado al tipo de práctica.

Palabras clave: Control de Infecciones; Infección Hospitalaria; Conocimientos, Actitudes y Práctica en Salud; Factores de Riesgo; Médicos; Perú (fuente: DeCS BIREME).

\section{Abstract}

Objectives. To determine level of knowledge and practices on the measures of prevention of infections associated with health care (HAl) and factors associated with in incoming resident physicians 2018 of a university of Lima, Peru. Methods. A validated questionnaire was applied to 171 resident physicians in a cross-sectional study. Variables studied were age, sex, time as a professional, previous training, origin, type of university of origin, among others. Results. It was observed that $52 \%$ are female, $49 \%$ had 2 or less years as a medical professional, $49 \%$ came from a university in the interior of the country, only $34 \%$ occupied a captive vacancy and $71.9 \%$ had previous training on $\mathrm{HAl}$ prevention measures. $83 \%$ of the incoming resident physicians studied showed a low level of knowledge about HAI prevention measures while $68.4 \%$ of the incoming resident physicians studied showed inappropriate practices on HAI prevention measures. When performing the multivariate analysis, it showed that entering a medicine specialty was associated with a lower risk of having low level of knowledge (OR: $0.32,95 \% \mathrm{Cl}: 0.11-0.93$ ). The bivariate analysis showed that the longest time as a professional was related to lower risk of having inappropriate practices, this being confirmed when performing the multivariate analysis (OR: $0.936,95 \% \mathrm{Cl}: 0.89-0.99)$. Conclusions. There is a low level of knowledge about HAI prevention measures and a high proportion with inappropriate practices in incoming residents 2018 on HAl prevention measures. It was observed that the type of specialty is a factor associated with the level of knowledge and the time of professional experience is a factor associated with the type of practice.

Keywords: Infection Control; Health Care Associated Infections; Health Knowledge, Attitudes, Practice; Risk Factors; Physicians; Peru (source: MeSH NLM). 


\section{INTRODUCCIÓN}

Las infecciones asociadas a la atención de salud (IAAS) también denominadas infecciones intrahospitalarias o infecciones asociadas a la atención sanitaria, son infecciones que se adquieren al interior de los establecimientos de salud, no habiendo estado presentes ni en incubación al momento del ingreso de los pacientes ${ }^{(1)}$. Las IAAS constituyen un problema de salud pública a nivel mundial ya que incrementan la morbilidad, la mortalidad, los costos de la atención (2) y comprometen la calidad de la atención de los establecimientos de salud, además que puedan generar potencialmente problemas médicos legales ${ }^{(3)}$.

En países de escasos y medianos recursos se ha observado prevalencias de IAAS que varían entre un 5,7\% a 19,1\%. El riesgo de IAAS en unidades de cuidados intensivos (UCl) se ha calculado que es 3 veces más en los países en vías de desarrollo comparado con los países desarrollados, este riesgo en $\mathrm{UCl}$ neonatal puede llegar a ser nueve veces mayor en países en desarrollo comparado con Estados Unidos de Norteamérica ${ }^{(4)}$. En el Perú, para el año 2015, el sistema de vigilancia epidemiológica reportó 6234 IAAS de un aproximado de 290 establecimientos de salud, siendo las principales IAAS notificadas las infecciones de herida operatoria (28\%), las infecciones del tracto urinario asociado a catéter urinario (20\%) y las neumonías asociadas a ventilación mecánica (20\%) ${ }^{(5)}$.

Las medidas de prevención de las IAAS se dividen en medidas generales denominadas precauciones estándar que incluyen la higiene de manos, el uso de equipo de protección personal, la higiene respiratoria, las prácticas de inyección segura, entre otras; y medidas específicas de prevención basadas según el mecanismo de transmisión de los microorganismos, que incluyen las precauciones por contacto, por gotitas y por aerosoles ${ }^{(6)}$. Las fallas en la aplicación de las medidas de prevención y control de infecciones no solo pueden afectar a los pacientes sino también afectar al personal de salud, manifestándose en infecciones adquiridas por dicho personal al interior del establecimiento de salud, como por ejemplo la tuberculosis (7), hepatitis B, influenza, entre otros.

A nivel mundial se han realizado diversos estudios que miden el nivel de conocimientos y prácticas sobre medidas de prevención de IAAS en personal de salud; sin embargo, estos estudios han sido realizados predominantemente en enfermería y estudiantes de ciencias de la salud. Los estudios en médicos residentes son escasos y más aún en residentes ingresantes.

En este contexto se planteó el objetivo de determinar el nivel de conocimientos y prácticas sobre la prevención de las infecciones asociadas a la atención de salud en médicos residentes ingresantes en una universidad de Lima, Perú.

\section{MÉTODOS}

\section{Tipo de estudio}

Se realizó un estudio observacional, analítico, de corte transversal.

\section{Población y muestra}

La población de estudio estuvo constituida por los médicos residentes ingresantes en el proceso de admisión al Programa de Segunda Especialización de Medicina de la Facultad de Medicina de la Universidad Nacional Mayor de San Marcos (UNMSM), en el año 2018. En el proceso de admisión 2018, postularon 1643 médicos, se ofertaron 611 vacantes e ingresaron 517 médicos residentes. Con la finalidad de asegurar que el tamaño muestral fuera suficiente para realizar los cálculos estadísticos, se calculó el tamaño muestral utilizando el software EPI INFO versión 7.2.2.6 considerando una frecuencia estimada del $60 \%$, un margen de error aceptable del $6 \%$ y un intervalo de confianza del 95\%. El tamaño muestral calculado fue de 171.

\section{Variables de estudio}

Las variables estudiadas fueron: a) Conocimientos de médicos residentes ingresantes sobre las medidas de prevención de las IAAS; b) Práctica referida de médicos residentes ingresantes sobre las medidas de prevención de las IAAS y; c) Los factores asociados al nivel de conoci- mientos y prácticas de médicos residentes ingresantes sobre las medidas de prevención de las IAAS (dimensiones: edad, sexo, tiempo como profesional, capacitaciones previas sobre control de infecciones, procedencia, tipo de universidad de procedencia del pregrado, especialidad y tipo de vacante).

\section{Instrumento y validación}

Se aplicó una encuesta estructurada previamente elaborada a partir de estudios previos $(8,9,10,11)$ y validada para medir el nivel de conocimientos y prácticas referidas, con las siguientes secciones: a) introducción; b) datos demográficos y ocupacionales; c) conocimientos sobre las medidas de prevención de las IAAS; y d) prácticas referidas sobre las medidas de prevención de las IAAS.

Para la validación del contenido, la encuesta se sometió al juicio de 10 expertos: 8 médicos con la especialidad de infectología (seis de los cuales tienen además experiencia en investigación, ética en investigación y salud pública), un salubrista y metodólogo con experiencia en validación de instrumentos y un médico gineco-obstetra con experiencia en control de infecciones. A dichos expertos se les invitó a participar en la validación del instrumento para lo cual se les entregó un resumen del protocolo de investigación, la encuesta propiamente dicha, los formatos de evaluación y un plazo de una semana para dicha evaluación. Se les pidió realizar una valoración del instrumento. Se aplicó el coeficiente " $V$ " de Aiken, resultando un valor de 0,97 que corresponde a una fuerte validez de contenido.

Para el análisis de confiabilidad se utilizó el coeficiente alfa de Cronbach obteniéndose para el instrumento global un valor de 0,64 lo que califica como de validez adecuada ${ }^{(8,11,12)}$. Al calcular el alfa de Cronbach para la sección de ítems sobre prácticas referidas se obtuvo un valor de 0,77 y para la sección de ítems sobre conocimientos se obtuvo un valor de 0,66.

\section{Valoración del conocimiento}

La sección que exploró el conocimiento sobre las medidas de prevención de las IAAS estuvo compuesta por 44 ítems (dentro de 11 preguntas), 32 
de los cuales con ítems tipo verdadero/ falso. Cada ítem puede adoptar el valor 1 si la respuesta es correcta, o el valor 0 si la respuesta es incorrecta. Así, los puntajes totales sobre los conocimientos se encuentran en un rango que va de 0 a 44. Los valores más elevados indicaron mayor conocimiento. Con relación al nivel de conocimiento $y$ otorgando una puntuación cualitativa a partir del puntaje total, se fijaron los siguientes niveles: conoce (cuando el puntaje obtenido es mayor de 33 respuestas correctas) y conoce poco (cuando el puntaje obtenido es menor o igual a 33 respuestas correctas). Esta división se realizó considerando un acierto mayor al $75 \%$ de los ítems de conocimiento ${ }^{(13)}$.

\section{Valoración de la práctica}

La sección que exploró las prácticas referidas sobre las medidas de prevención de las IAAS estuvo compuesta por 14 ítems, en los cuales se aplicó una escala de Likert, la cual expresa la frecuencia de una práctica, y que va desde 1 que equivale a nunca, hasta 5 que equivale a siempre. La práctica referida como adecuada correspondió a las prácticas catalogadas como "siempre" a la cual se le otorga un valor de 1, los otros enunciados ("nunca", "pocas veces", "algunas veces" y "casi siempre") correspondieron a prácticas deficientes y se les otorgó el valor de $0^{(14)}$. Con relación a la práctica, se otorgó una puntuación cualitativa a partir del puntaje total; así, se fijaron las siguientes categorías: práctica adecuada (cuando el puntaje global obtenido es mayor o igual a 10) y práctica deficiente (cuando el puntaje global es menor de 10). Esta división se realizó considerando un acierto mayor al $75 \%$ de los ítems de prácticas referidas.

\section{Análisis estadístico}

Los datos recolectados se ingresaron en una base de datos del programa Stata 14. En el análisis descriptivo, las variables cuantitativas, dependiendo de si tenían o no distribución normal, se presentaron a través de la media y desviación standard o la mediana y rango intercuartil. Para presentar las variables categóricas se utilizó frecuencias o porcentajes. En el análisis bivariado, para comparar variables continuas, debido a que no tenían distribución normal, se utilizó la prueba no paramétrica para dos muestras independiente o también llamada prueba de Mann Whitney (suma de rangos). Para comparar variables categóricas se utilizó la prueba de chi2. La asociación entre el puntaje obtenido de conocimientos y de prácticas fue analizada con la prueba de chi2. Para medir el riesgo y la asociación entre las variables se realizó un análisis de regresión logística múltiple. Se utilizó un $\alpha=0,05$ como nivel de significancia.

\section{Aspectos éticos}

Se mantuvo la confidencialidad de las respuestas y los datos personales de los participantes, pues la información recolectada no tuvo incidencia en la evaluación del examen de ingreso. Los médicos residentes fueron informados sobre el propósito y naturaleza del estudio, siendo su participación voluntaria y anónima. El protocolo de estudio fue presentado y aprobado por el Comité de Ética de la Facultad de Medicina de la Universidad Nacional Mayor de San Marcos.

\section{RESULTADOS}

De los 517 médicos residentes ingresantes a la UNMSM el año 2018, 171 respondieron la encuesta. El rango de edades de la población de estudio osciló entre 25 y 54 años, una tercera parte $(32,2 \%)$ de los residentes tenían entre 28 y 31 años de edad, seguido por los residentes mayores de 35 años de edad $(26,9 \%)$. La mayor proporción de residentes fueron de sexo femenino (52\%) y procedían de provincias $(55,6 \%)$, la mayoría de residentes tenía hasta 2 años de experiencia como profesional $(49,1 \%)$ y $21,1 \%$ más de 8 años de experiencia. El $49,1 \%$ cursó el pregrado de medicina en una universidad de provincias, mientras

Tabla 1. Características demográficas y ocupacionales de médicos residentes ingresantes al Programa de Segunda Especialización de Medicina de la Facultad de Medicina de la Universidad Nacional Mayor de San Marcos, 2018.

\begin{tabular}{|c|c|c|c|}
\hline Variable & Característica & $\begin{array}{c}\text { Frecuencia } \\
(n=171)\end{array}$ & Porcentaje \\
\hline \multirow[t]{4}{*}{ Edad (años) } & $24-27$ & 40 & $23,4 \%$ \\
\hline & $28-31$ & 55 & $32,2 \%$ \\
\hline & $32-35$ & 30 & $17,5 \%$ \\
\hline & $>35$ & 46 & $26,9 \%$ \\
\hline \multirow[t]{2}{*}{ Sexo } & Masculino & 82 & $48 \%$ \\
\hline & Femenino & 89 & $52 \%$ \\
\hline \multirow[t]{3}{*}{ Lugar de procedencia } & Lima & 72 & $42,1 \%$ \\
\hline & Provincias & 95 & $55,6 \%$ \\
\hline & Sin dato & 4 & $2,3 \%$ \\
\hline \multirow[t]{5}{*}{ Tiempo como profesional (años) } & $0-2$ & 84 & $49,1 \%$ \\
\hline & $2,1-5$ & 31 & $18,1 \%$ \\
\hline & $5,1-8$ & 16 & $9,4 \%$ \\
\hline & $>8$ & 36 & $21,1 \%$ \\
\hline & Sin dato & 4 & $2,3 \%$ \\
\hline \multirow[t]{4}{*}{$\begin{array}{l}\text { Universidad en la que curso el } \\
\text { pregrado según ubicación }\end{array}$} & Lima & 75 & $43,9 \%$ \\
\hline & Provincias & 84 & $49,1 \%$ \\
\hline & Extranjero & 11 & $6,4 \%$ \\
\hline & Sin dato & 1 & $0,6 \%$ \\
\hline \multirow[t]{2}{*}{ Vacante cautiva } & $\mathrm{Si}$ & 58 & $34 \%$ \\
\hline & No & 113 & $66 \%$ \\
\hline \multirow[t]{3}{*}{$\begin{array}{l}\text { Capacitación en medidas de } \\
\text { prevención de IAAS }\end{array}$} & $\mathrm{Si}$ & 123 & $71,9 \%$ \\
\hline & No & 47 & $27,5 \%$ \\
\hline & Sin dato & 1 & $0,6 \%$ \\
\hline
\end{tabular}


Tabla 2. Frecuencia de respuestas correctas sobre conocimientos de medidas de prevención de IAAS (precauciones estándar), en médicos residentes ingresantes de la Facultad de Medicina de la Facultad de Medicina de la Universidad Nacional Mayor de San Marcos, 2018.

\begin{tabular}{|c|c|c|}
\hline \multirow{2}{*}{ Preguntas } & \multicolumn{2}{|c|}{ Respuestas correctas } \\
\hline & $\mathbf{N}^{\circ}$ de residentes & Porcentaje \\
\hline \multicolumn{3}{|l|}{ Con relación a las infecciones asociadas a la atención de la salud: } \\
\hline $\begin{array}{l}\text { El ambiente (aire, agua, superficies inertes) es la mayor fuente de bacterias responsables para las IAAS } \\
(\mathrm{n}=170)\end{array}$ & 43 & 25,29 \\
\hline La Edad avanzada ó edad muy temprana incrementa el riesgo de IAAS ( $n=171)$ & 162 & 95,86 \\
\hline Los procedimientos invasivos incrementan el riesgo de IAAS $(n=171)$ & 167 & 97,66 \\
\hline $\begin{array}{l}\text { Los factores asociados a las prácticas para la prevención de las IAAS son muy importantes y modificables } \\
(\mathrm{n}=170)\end{array}$ & 164 & 96,47 \\
\hline \multicolumn{3}{|l|}{ Con relación a las precauciones estándar: } \\
\hline Incluyen las recomendaciones para la protección solo de los pacientes $(n=171)$ & 152 & 88,88 \\
\hline Incluyen las recomendaciones para la protección de los pacientes y los trabajadores de la salud $(n=171)$ & 169 & 98,83 \\
\hline Aplican para todos los pacientes $(n=171)$ & 136 & 79,53 \\
\hline Aplican solo para trabajadores de la salud quienes tienen contacto con fluidos corporales ( $n=170$ ) & 144 & 84,71 \\
\hline \multicolumn{3}{|l|}{ ¿Cuándo es recomendable la higiene de manos? } \\
\hline Antes o después de tener contacto con (o cuidar a) un paciente $(n=170)$ & 113 & 66,47 \\
\hline Antes y después de tener contacto con (o cuidar a) un paciente $(n=171)$ & 168 & 98,25 \\
\hline Entre contacto con pacientes $(n=171)$ & 151 & 88,3 \\
\hline Después de retirarse los guantes $(n=170)$ & 148 & 87,06 \\
\hline \multicolumn{3}{|l|}{ ¿Cuáles son las indicaciones para el uso de alcohol gel (en manos no sucias visiblemente)? } \\
\hline En lugar de una higiene de manos tradicional (30 segundos) $(n=171)$ & 120 & 70,18 \\
\hline En lugar de una higiene de manos con antisépticos (30 segundos) ( $n=171)$ & 80 & 46,78 \\
\hline En lugar de una higiene de manos quirúrgica (3 minutos) $(n=169)$ & 25 & 14,79 \\
\hline Una higiene de manos tradicional debería realizarse antes de la higiene de manos con alcohol gel $(n=170)$ & 79 & 46,47 \\
\hline \multicolumn{3}{|l|}{ Las precauciones estándar recomiendan el uso de guantes: } \\
\hline Para cada procedimiento $(n=171)$ & 10 & 8,85 \\
\hline Cuando hay un riesgo de contacto con sangre o fluidos corporales $(n=171)$ & 157 & 91,81 \\
\hline Cuando hay un riesgo de una cortadura $(n=171)$ & 146 & 85,38 \\
\hline Cuando el trabajador de la salud tiene una lesión cutánea $(n=171)$ & 154 & 90,06 \\
\hline \multicolumn{3}{|l|}{$\begin{array}{l}\text { Cuando hay riesgo de salpicaduras con sangre y fluidos corporales, los trabajadores de salud deben } \\
\text { usar: }\end{array}$} \\
\hline Solo mascarilla $(n=171)$ & 167 & 97,66 \\
\hline Solo lentes de protección $(n=171)$ & 167 & 97,66 \\
\hline Solo mandil o bata $(n=169)$ & 166 & 98,22 \\
\hline $\begin{array}{l}\text { Mascarilla, lentes protectores y mandil o bata } \\
(\mathrm{n}=171)\end{array}$ & 168 & 98,25 \\
\hline \multicolumn{3}{|l|}{ Con relación al uso de equipo de protección personal (EPP) } \\
\hline El EPP debe usarse solo cuando haya contacto con sangre $(n=171)$ & 152 & 88,88 \\
\hline El EPP es exclusivamente adecuado para el personal de laboratorio y limpieza para su protección $(n=171)$ & 143 & 83,63 \\
\hline Los guantes deben cambiarse entre diferentes procedimientos en el mismo paciente $(n=171)$ & 151 & 88,3 \\
\hline El uso de EPP disminuye el riesgo de adquirir infecciones ocupacionales $(n=170)$ & 164 & 96,47 \\
\hline \multicolumn{3}{|l|}{ Con relación a la eliminación y lesiones por objetos punzo cortantes: } \\
\hline Las agujas usadas deben volver a taparse después del uso para evitar lesiones $(n=171)$ & 119 & 69,59 \\
\hline Las agujas usadas deben doblarse después del uso para evitar lesiones $(n=171)$ & 152 & 88,88 \\
\hline Las lesiones punzo cortantes deben ser manejadas sin informar $(n=170)$ & 166 & 97,65 \\
\hline Las lesiones con agujas son menos comunes en la práctica general $(n=171)$ & 152 & 88,88 \\
\hline
\end{tabular}


que el 43,9\% en una universidad de Lima, solo el 6,4\% realizó el pregrado en el extranjero. El 34\% de residentes ingresantes ocupó una vacante cautiva, y el 71,9\% recibió una capacitación en medidas de prevención de las IAAS. Tabla 1.

Cuando se analizó la distribución de los médicos residentes según región de procedencia, la mayoría $42,11 \%$ era de Lima seguido por los que procedían de Cusco y Piura (7,02\% cada uno), Junín $(6,43 \%)$, Ica y Ucayali $(6,1 \%$ cada uno). La distribución de médicos residentes encuestados, según especialidad de residencia, mostró que el mayor porcentaje eran de la especialidad de gineco-obstetricia $(15,8 \%)$, seguido por pediatría $(11,7 \%)$. Seis especialidades concentraron el $50 \%$ de los médicos residentes: gineco-obstetricia, pediatría, medicina física y rehabilitación, anestesiología, patología clínica y psiquiatría.

Al analizar el nivel de conocimientos de los médicos residentes encuestados se observó que la mayoría conocía poco sobre las medidas de prevención de las IAAS, en comparación con los que sí las conocía ( $83 \%$ versus 17\%). Cuando se exploró el nivel de práctica referida de los médicos residentes encuestados, se observó que la mayoría $(68,4 \%)$ tenían prácticas deficientes sobre las medidas de prevención de las IAAS.

La evaluación del nivel de conocimientos incluyó diversos aspectos de las medidas de prevención de IAAS como las precauciones estándar, higiene de manos, uso de equipo de protección personal y eliminación y lesiones por objetos punzo cortantes, observándose poco conocimiento en algunas indicaciones del uso de alcohol gel $(14,79 \%)$ y en la higiene de manos $(66,47 \%)$ y con relación a la eliminación de objetos punzo cortantes (69,59\%). Tabla 2.

La evaluación de las prácticas referidas sobre las medidas de prevención de IAAS incluyó aspectos como la higiene de manos, el uso de guantes, batas, mascarilla, protección ocular, uso de respirador N95. Entre los hallazgos más relevantes se observó con relación a la práctica de higiene de manos o uso de solución hidroalcohólica, que aproximadamente solo un tercio de los encuestados señalo que siempre realizaba la higiene de manos antes y después de atender un paciente, y solo un tercio realizaba esta práctica después de tener contacto con el paciente o su entorno. Solo el 50,8\% utilizaba siempre respirador $\mathrm{N} 95$ al atender un paciente con hemoptisis, el $44,1 \% \%$ siempre utilizaba mascarilla al atender un paciente con sospecha de influenza. Tabla 3.

Tabla 3. Frecuencia de respuestas correctas sobre prácticas referidas de medidas de prevención de IAAS, en médicos residentes ingresantes de la Facultad de Medicina de la Facultad de Medicina de la Universidad Nacional Mayor de San Marcos, 2018.

\begin{tabular}{|c|c|c|}
\hline \multirow[b]{2}{*}{ Preguntas } & \multicolumn{2}{|c|}{ Respuestas correctas } \\
\hline & $\begin{array}{l}\mathrm{N}^{\circ} \text { de } \\
\text { residentes }\end{array}$ & Porcentaje \\
\hline $\begin{array}{l}\text { 1.- Frecuencia con que realiza la higiene de manos o usa solución } \\
\text { hidroalcohólica antes y después de atender a un paciente }(n=171)\end{array}$ & 54 & 31,58 \\
\hline $\begin{array}{l}\text { 2.- Frecuencia con que realiza la higiene de manos o usa solución } \\
\text { hidroalcohólica antes de realizar una técnica limpia/aséptica } \\
(n=170)\end{array}$ & 102 & 60 \\
\hline $\begin{array}{l}\text { 3.- Frecuencia con que realiza la higiene de manos o usa solución } \\
\text { hidroalcohólica después de tener contacto con el paciente o su } \\
\text { entorno próximo } \\
(n=170)\end{array}$ & 55 & 32,35 \\
\hline
\end{tabular}

4.- Frecuencia con que realiza la higiene de manos si existe el riesgo o la certeza de haber tocado sangre, fluidos biológicos, secreciones $u$ 141 82,46 objetos contaminados $(n=171)$

5.- Frecuencia con que realiza la higiene de manos o usa solución hidroalcohólica antes y después de realizar un procedimiento 111 65,68 $(n=169)$

6.- Frecuencia con que realiza la higiene de manos o usa solución hidroalcohólica después de retirarse los guantes $(n=171)$

74

43,27

7.- Frecuencia con que utiliza guantes al realizar extracciones de muestra sanguíneas venosas $(n=168)$

8.- Frecuencia con que utiliza guantes al realizar la curación de una herida $(n=170)$

9.- Frecuencia con que usa batas para la protección de la piel y para evitar ensuciarse la ropa durante las actividades en las que se pueden producir salpicaduras de sangre, fluidos biológicos, secreciones o excreciones $(n=169)$

10.- Frecuencia con la que se coloca la bata, mascarilla y protección ocular cuando existe riesgo de salpicaduras al realizar una técnica o procedimiento $(n=170)$

\begin{tabular}{lcc}
$\begin{array}{l}\text { 11.- Frecuencia con que ha vuelto a encapsular una aguja antes de } \\
\text { depositarla en el contenedor para material biopeligroso }(n=171)\end{array}$ & 40,39 \\
\hline $\begin{array}{l}\text { 12.- Frecuencia con que utiliza respirador N95 al atender un paciente } \\
\text { con hemoptisis }(n=171)\end{array}$ & 57,88 \\
$\begin{array}{l}\text { 13.- Frecuencia con que utiliza equipo de protección personal } \\
\text { (guantes, mascarilla, bata) al manipular paciente colonizado/ } \\
\text { infectado con microorganismo resistente ( } n=171)\end{array}$ & 91 & 53,22 \\
\hline $\begin{array}{l}\text { 14.- Frecuencia con que utiliza una mascarilla al atender un paciente } \\
\text { con sospecha de influenza } \\
\text { (n=170) }\end{array}$ & 75 & 44,12 \\
\end{tabular}


En el análisis bivariado de la relación entre el nivel de conocimientos sobre las medidas de prevención de IAAS con las variables edad, sexo, departamento de procedencia, tiempo como profesional, universidad en la que curso el pregrado según ubicación, vacante cautiva, antecedente de capacitación sobre medidas de prevención de IAAS y tipo de especialidad, se determinó que las únicas variables que estaban relacionadas fueron la vacante cautiva $(p=0,037)$ y el tipo de especialidad ( $p=0,032$ ). Tabla 4.

En el análisis bivariado de la relación entre el nivel de prácticas sobre las medidas de prevención de IAAS con las variables edad, sexo, departamento de procedencia, tiempo como profesional, universidad en la que curso el pregrado según ubicación, vacante cautiva, antecedente de capacitación sobre medidas de prevención de IAAS y tipo de especialidad, se determinó que la única variable relacionada fue el tiempo como profesional expresado en años ( $p=0,0125)$, teniendo una mediana de 4,5 años en aquellos residentes que tenían prácticas adecuadas en comparación con los residentes que tenían prácticas inadecuadas con una mediana de 2 años. Tabla 5.

Al realizar el análisis multivariado y comparar la variable especialidad médica con nivel de conocimientos, se observó que los médicos residentes ingresantes a una especialidad médica tenían $68 \%$ menos riesgo de tener poco conocimiento sobre medidas de prevención de IAAS en comparación con los médicos residentes ingresantes a una especialidad quirúrgica (OR: 0,32, IC: 0,11-0,93). No se encontró asociación entre la variable vacante cautiva y el nivel de conocimientos (OR: 1,74, IC 0,43-7,01). En el análisis multivariado, el tiempo de experiencia profesional se encontró asociado con una menor proporción de prácticas inadecuadas (OR:

Tabla 4. Nivel de conocimientos sobre medidas de prevención de IAAS según edad, sexo, procedencia, tiempo como profesional, universidad, vacante cautiva y antecedente de capacitación, en médicos residentes ingresantes de la Facultad de Medicina de la Facultad de Medicina de la Universidad Nacional Mayor de San Marcos, 2018. Análisis bivariado.

\begin{tabular}{|c|c|c|c|c|}
\hline \multirow{2}{*}{ Variable } & \multirow{2}{*}{ Característica } & Conoce & Conoce poco & \multirow{2}{*}{$\mathbf{p}$} \\
\hline & & $\mathbf{N}^{\circ}(\%)$ & $\mathbf{N}^{\circ}(\%)$ & \\
\hline \multirow{2}{*}{ Edad } & $<30$ años & $16(21,92 \%)$ & $57(78,08 \%)$ & \multirow{2}{*}{$0,136^{*}$} \\
\hline & $\geq 30$ años & $13(13,27 \%)$ & $85(86,73 \%)$ & \\
\hline \multirow{2}{*}{ Sexo } & Masculino & $16(19,51 \%)$ & $66(80,49 \%)$ & \multirow[t]{2}{*}{$0,393 * *$} \\
\hline & Femenino & $13(14,61 \%)$ & $76(85,39 \%)$ & \\
\hline \multirow{2}{*}{ Departamento de procedencia } & Lima & $13(18,06 \%)$ & $59(81,94 \%)$ & \multirow{2}{*}{$0,745^{* *}$} \\
\hline & Provincias & $16(16,16 \%)$ & $83(83,84 \%)$ & \\
\hline $\begin{array}{l}\text { Tiempo como profesional } \\
\text { en años (mediana y rango } \\
\text { intercuartil) }\end{array}$ & & $2(1-5)$ & $3(1-8)$ & $0,1311^{*}$ \\
\hline \multirow{3}{*}{$\begin{array}{l}\text { Universidad en la que curso el } \\
\text { pregrado según ubicación }\end{array}$} & Lima & $15(20 \%)$ & $60(80 \%)$ & \multirow{3}{*}{$0,630 * *$} \\
\hline & Provincias & $12(14,29 \%)$ & $72(85,71 \%)$ & \\
\hline & Extranjero & $2(18,18 \%)$ & $9(81,82 \%)$ & \\
\hline \multirow{2}{*}{ Vacante cautiva } & $\mathrm{Si}$ & $5(8,62 \%)$ & $53(91,38 \%)$ & \multirow[t]{2}{*}{$0,037^{* *}$} \\
\hline & No & $24(21,24 \%)$ & $89(78,76 \%)$ & \\
\hline \multirow{2}{*}{$\begin{array}{l}\text { Antecedentes de capacitación } \\
\text { (en medidas de prevención de } \\
\text { IAAS) }\end{array}$} & $\mathrm{Si}$ & $21(17,07 \%)$ & $102(82,93 \%)$ & \multirow[t]{2}{*}{$0,994^{* *}$} \\
\hline & No & $8(17,02 \%)$ & $39(82,98 \%)$ & \\
\hline \multirow{2}{*}{ Tipo de especialidad } & Médica & $24(21,43 \%)$ & $88(78,57 \%)$ & \multirow{2}{*}{$0,032 * *$} \\
\hline & Quirúrgica & $5(8,47 \%)$ & $54(91,53 \%)$ & \\
\hline
\end{tabular}

* Prueba de Mann Whitney

** Prueba de $\mathrm{Chi}^{2}$
0,936, IC 0,89-0,99). Finalmente, al comparar el nivel de conocimientos con las prácticas referidas sobre las medidas de prevención de IAAS en los médicos residentes ingresantes, no se encontró asociación entre ambas variables $(p=0,612)$.

\section{DISCUSIÓN}

Las infecciones asociadas a la atención de la salud (IAAS) son un problema de salud pública mundial dado que incrementan la morbilidad, la mortalidad, los costos hospitalarios y afectan la calidad de la atención ${ }^{(1,2)}$.

A nivel mundial se han realizado múltiples estudios sobre los conocimientos, actitudes y prácticas de medidas de prevención de IAAS en personal de salud, así por ejemplo dichos estudios predominan en personal de enfermería, estudiantes en ciencias de la salud y en menor proporción en personal médico. Existen pocos estudios sobre conocimientos y prácticas en médicos residentes a nivel mundial. Estos estudios incluyen temas como la higiene de manos, precauciones estándar y precauciones de aislamiento ${ }^{(4,8,9)}$.

En este estudio se observó que el 83\% de los residentes ingresantes 2018 de la UNMSM conocían poco sobre las medidas de prevención de las IAAS mientras que el $64 \%$ tenían prácticas deficientes sobre las medidas de prevención de las IAAS. Estos resultados son preocupantes y deben llevar a replantear el reforzamiento de algunos temas vinculados a la prevención y control de infecciones en los planes de estudios de las universidades tanto a nivel de pregrado como del postgrado.

Estudios en diferentes continentes han reportado resultados similares al nuestro, como por ejemplo un estudio realizado en México que exploró sobre los conocimientos y aptitudes para la aplicación de las precauciones de aislamiento en un hospital pediátrico de tercer nivel, en el cual el 36\% de su población de estudio eran médicos residentes, demostrando que el $75 \%$ de los encuestados tenían conocimientos deficientes, un $17 \%$ conocimiento regular y un $8 \%$ conocimiento adecuado sobre las precauciones de aislamiento ${ }^{(9)}$. 
Tabla 5. Nivel de práctica referida sobre medidas de prevención de IAAS según edad, sexo, procedencia, tiempo como profesional, universidad, vacante cautiva y antecedente de capacitación. en médicos residentes ingresantes de la Facultad de Medicina de la Facultad de Medicina de la Universidad Nacional Mayor de San Marcos, 2018. Análisis bivariado.

\begin{tabular}{|c|c|c|c|c|}
\hline \multirow[t]{2}{*}{ Variable } & \multirow[t]{2}{*}{ Característica } & $\begin{array}{c}\text { Práctica } \\
\text { adecuada }\end{array}$ & $\begin{array}{c}\text { Práctica } \\
\text { deficiente }\end{array}$ & \multirow[t]{2}{*}{$\mathbf{p}$} \\
\hline & & $\mathbf{N}^{\circ}(\%)$ & $\mathbf{N}^{\circ}(\%)$ & \\
\hline \multirow{2}{*}{ Edad } & $<30$ años & $20(27,4)$ & $53(72,6)$ & \multirow{2}{*}{$0,310 * *$} \\
\hline & $\geq 30$ años & $34(34,7)$ & $64(65,3)$ & \\
\hline \multirow{2}{*}{ Sexo } & Masculino & $22(26,8)$ & $60(73,2)$ & \multirow{2}{*}{$0,200 * *$} \\
\hline & Femenino & $32(35,9)$ & $57(64,1)$ & \\
\hline \multirow{2}{*}{ Departamento de procedencia } & Lima & $26(36,1)$ & $46(63,9)$ & \multirow{2}{*}{$0,277^{* *}$} \\
\hline & Provincias & $28(28,3)$ & $71(71,7)$ & \\
\hline Tiempo como profesional en años (mediana y rangos intercuartiles) & № de años & $4,5(\mathrm{RI}: 2-10)$ & $2(\mathrm{Rl}: 1-6)$ & $0,0125^{*}$ \\
\hline \multirow{3}{*}{ Universidad en la que curso el pregrado según ubicación } & Lima & $27(36)$ & $48(64)$ & \multirow{3}{*}{$0,483^{* *}$} \\
\hline & Provincias & $23(27,4)$ & $61(72,6)$ & \\
\hline & Extranjera & $3(27,3)$ & $8(72,7)$ & \\
\hline \multirow{2}{*}{ Vacante cautiva } & $\mathrm{Si}$ & $21(36,2)$ & $37(63,8)$ & \multirow{2}{*}{$0,351 * *$} \\
\hline & No & $33(29,2)$ & $80(70,8)$ & \\
\hline \multirow{2}{*}{ Antecedentes de capacitación en medidas de prevención de IAAS } & $\mathrm{Si}$ & $40(32,5)$ & $83(67,5)$ & \multirow{2}{*}{$0,732 * *$} \\
\hline & No & $14(29,8)$ & $33(70,2)$ & \\
\hline \multirow{2}{*}{ Tipo de especialidad } & Médicas & $34(30,4)$ & $78(69,6)$ & \multirow{2}{*}{$0,636 * *$} \\
\hline & Quirúrgicas & $20(33,9)$ & $39(66,1)$ & \\
\hline
\end{tabular}

* Prueba de Mann Whitney

** Prueba de $\mathrm{Chi}^{2}$

En Francia, en un hospital universitario se evaluó los conocimientos y actitudes relacionadas a las precauciones de aislamiento. Del total de médicos que respondieron el cuestionario, el $46 \%$ eran médicos residentes, los resultados demostraron que el nivel de conocimientos sobre los diferentes tipos de precauciones de aislamiento en los médicos residentes era bajo, así por ejemplo el nivel de respuestas correctas sobre las precauciones aéreas fue del $17,6 \%$, el nivel de respuestas correctas sobre precauciones por gotitas fue del $11,8 \%$ y el nivel de respuestas correctas sobre precauciones por contacto fue del $56,9 \%{ }^{(15)}$.

Un estudio realizado en una universidad venezolana a fines del año 2018 en médicos residentes, exploró el nivel de conocimientos sobre IAAS, precauciones universales e higiene de manos. Si bien el 56,87\% aprobó la encuesta de conocimientos, los menores niveles de conocimientos fueron sobre la higiene de manos y las precauciones universales ${ }^{(16)}$.
A diferencia de nuestros resultados, Askarian exploró los conocimientos, actitudes y prácticas relacionados a las precauciones estándar de cirujanos, médicos, residentes de cirugía y residentes de medicina de Irán. El estudio incluyo 78 médicos asistentes y 77 médicos residentes, encontrando que los conocimientos de los médicos residentes de cirugía y de medicina tenían un buen nivel de conocimientos (mediana de 7 , sobre un puntaje máximo de 9) pero el nivel de prácticas no fueron las adecuadas tanto para los médicos residentes de medicina como para los residentes de cirugía (17).

En el año 2012 se publicó un estudio que evaluó las prácticas de control de infecciones en trabajadores de salud de un hospital de Perú, dicho estudio incluyó a médicos, residentes, enfermeras e internos de medicina, reportando que solo un $14,5 \%$ tenían prácticas adecuadas de control de infecciones y que solo el $23,6 \%$ se lavaron las manos antes y después de tener contacto con un paciente resultado similar a lo encontrado en la presente investigación ${ }^{(18)}$.

Es importante resaltar el poco conocimiento mayoritariamente observado entre los encuestados del presente estudio, sobre todo en aspectos fundamentales de la prevención de las IAAS; por ejemplo, el desconocimiento que el ambiente no es la principal fuente de bacterias responsables de las IAAS o que el uso de alcohol gel no sustituye a una higiene de manos quirúrgica.

En el estudio se encontró un elevado porcentaje $(71,9 \%)$ de médicos residentes que refirió haber recibido capacitación sobre medidas de prevención de IAAS; sin embargo, el nivel de conocimiento fue deficiente en un gran porcentaje. Esto puede obedecer a un insuficiente tiempo de capacitación recibido o un currículo con insuficiente contenido sobre las medidas de prevención de IAAS. Es oportuno mencionar que en la encuesta no se preguntó sobre el tipo o modalidad de capacitación entre los mé- 
dicos residentes que refirieron haberla recibido.

Lo observado en cuanto a las prácticas referidas entre los encuestados revelan, de igual forma, prácticas inadecuadas en aspectos fundamentales de la prevención de las IAAS; por ejemplo, solo una tercera parte de los encuestados realizó siempre la higiene de manos o usa solución hidroalcohólica antes y después de atender a un paciente.

En la investigación se identificó que el tipo de especialidad al cual ingresó el residente es un factor asociado al nivel de conocimientos, es así que los residentes que ingresaron a una especialidad de medicina tenían $68 \%$ menos riesgo de tener poco conocimiento sobre las medidas de prevención de las IAAS en comparación con los médicos residentes que ingresaron a especialidades quirúrgicas.

En el estudio se identificó que el tiempo de experiencia profesional fue un factor asociado a la práctica referida de los médicos residentes ingresantes 2018 de la UNMSM sobre las medidas de prevención de las IAAS. Los médicos residentes con mayor tiempo de experiencia profesional tuvieron 6,4\% menor probabilidad de tener prácticas inadecuadas.

En general los resultados de los estudios a nivel internacional para identificar factores asociados al nivel de conocimientos sobre medidas de prevención de IAAS han mostrado resultados diversos; así por ejemplo, en Georgia, Butsashvili identificó mediante análisis multivariado que los factores asociados al nivel de conocimientos fueron la edad $(\geq$ de 35 años de edad tenían mayor nivel de conocimientos), la posición (los médicos mostraron mayor nivel de conocimientos que las enfermeras) y el haber recibido capacitación sobre precauciones estándar ${ }^{(19)}$. De igual forma Díaz-Peña y colaboradores identificaron que tener una antigüedad igual o mayor a 5 años (OR 2,82 , IC $1,15-6,88$ ) y no haber recibido capacitación en el último año (OR 10,5, IC $4,14-26,6)$ se asociaron a conocimientos deficientes sobre precauciones de aislamiento, observando que el no haber recibido capacitación en el último año era un factor de riesgo para una aptitud deficiente en la aplicación de las precauciones de aislamiento (OR 4,2, IC 1,93-9,14); sin embargo, la antigüedad laboral en el hospital no fue un factor asociado a la aptitud en la aplicación de las precauciones de aislamiento ${ }^{(9)}$.

Un aspecto no abordado en el presente estudio y que podría explicar en parte el nivel de conocimientos y las prácticas entre los encuestados, es la presencia de contenidos sobre medidas de prevención de infecciones en los cursos principales de medicina y cirugía de los planes curriculares de las diversas universidades de procedencia de los residentes ingresantes. Este aspecto amerita ser estudiado a través de estudios cualitativos que exploren e identifiquen la presencia de contenidos temáticos sobre medidas de prevención de IAAS, además de estudiar la calidad de dichos contenidos, sobre todo basados en evidencia científica.

Entre las limitaciones se señala que la medición de las prácticas no se ha realizado de forma directa a través de la observación dadas las complicaciones logísticas que ello implicaba, por la numerosa cantidad de sedes docentes para todas las especialidades; por ello se recurrió a la medición de las prácticas referidas utilizando una escala de Likert. Otra limitación fue que no todos los residentes de todas las especialidades participaron en forma homogénea; por ejemplo, en el estudio, el $40 \%$ de los médicos residentes que contestaron la encuesta procedían de 4 especialidades (gineco-obstetricia, pediatría, medicina física y rehabilitación y anestesiología).

La investigación realizada proporciona un instrumento de medición validado y abre el camino para futuras investigaciones que permitan evaluar el impacto de diversas intervenciones y modalidades de capacitación y entrenamiento en el tema de prevención de IAAS. La evidencia generada permitirá a su vez sensibilizar a las autoridades universitarias sobre la importancia del reforzamiento a nivel de posgrado sobre aspectos y contenidos de prevención y control de las infecciones asociadas a la atención de salud.

\section{REFERENCIAS BIBLIOGRÁFICAS}

1. Organización Panamericana de la Salud. Vigilancia Epidemiológica de las Infecciones Asociadas a la Atención de Salud. Washington DC: OPS. 2010: 13.
2. Organización Panamericana de la Salud. En: Salvatierra-González MR (ed): Costo de la infección nosocomial en nueve paises de América Latina. Washington DC: OPS. 2003: 181.

3. Organización Panamericana de la Salud. Infecciones hospitalarias. Legislación en América Latina. Washington DC: OPS. 2007: 1-2.

4. Allegranzi B, Nejad S, Combescure C, Graafmans W, Attar H, Donaldson L, et al. Burden of endemic health-care associated infection in developing countries: systematic review and meta-analysis. Lancet. 2011 Jan 15;377(9761):228-41. DOI: 10.1016/S0140-6736(10)61458-4

5. Quispe Z. Incidencia de infecciones asociadas a la atención de la salud en establecimientos de salud con internamiento en el Perú, 2015. Boletin Epidemiológico del Perú. 2016;25(37):809-813.

6. Siegel JD, Rhinehart E, Jackson M, Chiarello L. Guideline for Isolation Precautions: Preventing Transmission of Infectious Agents in Health Care Settings. Am J Infect Control. 2007 Dec;35(10 Suppl 2):S65-164. DOI: 10.1016/j.ajic.2007.10.007

7. Soto G, Chávez AM, Arrasco J, Yagui M. Tuberculosis en trabajadores de salud en el Perú, 2013-2015. Rev Peru Med Exp Salud Publica. Oct-Dec 2016;33(4):607-615. DOI: 10.17843/ rpmesp.2016.334.2542

8. Tavolacci MP, Ladner J, Bailly L, Merle V, Pitrou I, Czernichow P. Prevention of Nosocomial Infection and Standard Precautions: Knowledge and source of information among healthcare students. Infect Control Hosp Epidemiol. 2008 Jul;29(7):642-7. DOI: 10.1086/588683

9. Diaz R, Irissont JA, Barrera JC. Grado de conocimiento y aptitud para la aplicación de las precauciones de aislamiento en un hospital pediátrico de tercer nivel. Gac Med Mex. Sep-Oct 2015;151(5):567-75.

10. Krubrani A, Albesher M, Alkahtani A, Alamri F, Alshamrani M, Masuadi E. Knowledge and information sources on standard precautions and infection control of health sciences students at King Saud bin Abdulaziz University for Health Sciences, Saudi Arabia, Riyadh. J Infect Public Health. Jul-Aug 2018;11(4):546-549. DOI: 10.1016/j. jiph.2017.10.013

11. Castillo J. Medidas de prevención de la transmisión de infecciones nosocomiales y de bioseguridad: percepción de los estudiantes del grado de enfermeria (Tesis Doctoral). Extremadura, España: Universidad de Extremadura. 2015: 500

12. Brevidelli MM, Cianciarullo TI. Psychosocial and organizational factors relating to adhrence to standard precautions. Rev Saude Publica. 2009 Dec;43(6):907-16. DOI: 10.1590/s003489102009010700001

13. Olivera A, Cardoso C, Mascarenha D. Conocimiento y comportamiento de los profesionales de un centro de terapia intensiva en relación a la adopción de las precauciones de contacto. Rev Latino-am Enfermagem. 2009 setembrooutubro;17(5):625-631. DOI: 10.1590/S010411692009000500005

14. Colet P, Cruz J, Alotaibi K, Colet M, Islam S. Compliance with standard precautions among baccalaureate nursing students in a Saudi university: A self-report study. J Infect Public Health. Jul-Aug 2017;10(4):421-430. DOI: 10.1016/j. jiph.2016.06.005

15. Moriceau G, Gagneux-Brunon A, Gagnaire J, Mariat C, Lucht F, Berthelot P, et al. Preventing healthcare-associated infections: Residents 
and attending physicians need better training in advance isolation precautions. Med Mal Infect. 2016 Feb;46(1):14-9. DOI: 10.1016/j.medmal.2015.11.001

16. Guevara A, Figuera A. Conocimiento sobre infecciones asociadas a la atención de la salud en residentes de postgrado de una universidad venezolana. Kasmera. 2018;46(2), 127-138
17. Askarian M, McLaws M, Meylan M. Knowledge, attitude, and practices related to standard precautions of surgeons and physicians in university-affiliated hospitals of Shiraz, Iran. Int J Infect Dis. 2007 May;11(3):213-9. DOI: 10.1016/j.jij.2006.01.006

18. Yagui M, Tarqui C, Sanabria H, Encarnación E. Evaluación de las prácticas de control de infecciones de los trabajadores de la salud en un hospital de Lima Metropolitana, durante la epidemia de Influenza A H1N1. Revista de Salud Pública. 2012;14(2), 271-281.

19. Butsashvili M, Kamkamidse G, Umikashvili L, Gvinjilia L, Kankadze K, Berdzuli N. Knowledge of health care-associated infections among Georgian obstetricians and gynecologists. J Infect Dev Ctries. 2010 Jun 3;4(5):329-33. DOI: 10.3855/jidc.526 